\title{
Interactions Between Solanaceous Crops and 'Candidatus Liberibacter solanacearum' Haplotypes in Relation to Infection and Psyllid Survival on the Hosts
}

\author{
Fekede Workneh, ${ }^{\dagger}$ Li Paetzold, and Charles M. Rush \\ Texas A\&M AgriLife Research, Bushland, TX 79012
}

\begin{abstract}
'Candidatus Liberibacter solanacearum' (Lso), transmitted by the potato psyllid (Bactericera cockerelli), is the putative causal agent of potato zebra chip disease. The bacterial pathogen infects a wide range of solanaceous plants (both wild and cultivated species), among which are peppers, potatoes, and tomatoes. Currently there are two commonly detected, genetically distinct haplotypes of Lso (A and B) identified from potatoes in the United States. To determine whether there are interactions between Lso haplotypes and different solanaceous hosts, experiments were conducted in the greenhouse in which pepper, potato, and tomato plants were infested with psyllids carrying Lso A, B, or an A and $B$ mix $(\mathrm{AB})$ or with psyllids free of Lso. Host plants were grown in pots in cages on the greenhouse benches and infested with six psyllids per plant. In addition, eight pepper cultivars were similarly infested for deeper understanding of host-haplotype interactions. Approximately 7 weeks after infestation, adult psyllids in each cage were counted to determine the impact of Lso haplotype-host interactions

of Lso copiously reproduced on all three hosts, and leaf tissue from each plant tested positive for the respective Lso except those infested with Lso-negative psyllids. However, psyllids carrying Lso A did not survive on peppers but survived and abundantly reproduced on potatoes and tomatoes. In addition, samples from peppers infested with psyllids carrying Lso A tested negative for Lso. However, peppers infested with individual psyllids carrying Lso $\mathrm{AB}$ tested positive for Lso A, indicating that the presence of B may be required for infection by Lso A and psyllid survival on peppers. The different pepper cultivars infested with psyllids carrying Lso A showed similar results to the haplotype-host interaction tests, suggesting that cultivar may not be a factor in Lso A-pepper host interactions. Results from these studies suggest that Lso A may affect host selection by psyllids either for nutrition or laying of eggs. Mechanisms involved in preventing psyllid reproduction on peppers, once identified, will have significant implications for potential psyllid management.
\end{abstract} on psyllid survival and plants were sampled and tested molecularly for Lso. Individual psyllids carrying haplotypes $\mathrm{B}$ or $\mathrm{AB}$ and those free
Keywords: pathogen diversity, prokaryotes, vegetables
'Candidatus Liberibacter solanacearum' (Lso), which is transmitted by the potato psyllid (Bactericera cockerelli), is the putative causal agent of potato zebra chip disease (Hansen et al. 2008; Liefting et al. 2008, 2009; Munyaneza et al. 2007). The disease was first reported in Saltillo, Mexico, in the mid-1990s (Secor and Rivera 2004) and in South Texas in 2000 (Secor et al. 2006). Zebra chip is primarily characterized by internal tuber browning and necrosis, in which the tuber turns dark when sliced and fried, affecting the marketability of fried potato products such as chips and French fries (Munyaneza et al. 2007; Rashed et al. 2013; Rush et al. 2015). During the early stages of potato zebra chip disease discovery, growers in Texas incurred substantial losses to the disease (Rosson 2006) because proper management practices could not be implemented as a result of the lack of knowledge of the disease etiology. Potato zebra chip disease is currently prevalent in much of the U.S. potato production region, including the Pacific Northwest (Idaho, Oregon, and Washington; Crosslin et al. 2012a, b; Wen et al. 2009), in New Zealand (Liefting et al. 2008, 2009), and in several Latin American countries (Munyaneza et al. 2012b; Rehman et al. 2010; Secor and Rivera 2004).

${ }^{\dagger}$ Corresponding author: F. Workneh; F-workneh@tamu.edu

Funding: This work was supported by the Texas Insect-Vector Disease Research Initiative and the U.S. Department of Agriculture National Institute of Food and Agriculture Zebra Chip Specialty Crop Research Initiative (grant 2009-51181-20176).

The author(s) declare no conflict of interest.

Accepted for publication 12 July 2019.

(C) 2020 The American Phytopathological Society
Currently there are two widely detected haplotypes of Lso (A and B) in the United States (Lin and Gudmestad 2013; Lin et al. 2012; Wen et al. 2009, 2013). A third haplotype (F) was recently identified from a single tuber, which originated from southern Oregon (Swisher Grimm and Garczynski 2019). To date, haplotype A is the only Lso type identified in New Zealand (Nelson et al. 2011; Thompson et al. 2015). Lso, transmitted by other psyllid species, has also been reported to infect nonsolanaceous crops such as carrots and celery in Europe and has been given haplotype names from $\mathrm{C}$ to $\mathrm{E}$ and $\mathrm{U}$ (Alfaro-Fernández et al. 2012a, b; European and Mediterranean Plant Protection Organization 2012; Haapalainen et al. 2018; Munyaneza et al. 2010, 2012a, b). A detailed review is presented by Vereijssen et al. (2018).

Each Lso haplotype can be detected individually in psyllids and plant samples or both can be detected together in the same plant sample or psyllid as an A and B mix, referred to hereafter as "AB" (Wen et al. 2013; Workneh et al. 2018). Recent surveys of potato fields and native vegetation in the southwestern United States showed haplotype A to be more prevalent than B (Workneh et al. 2018). Two recent studies indicate that Lso B produces more severe symptoms in both potatoes and tomatoes than Lso A (Mendoza-Herrera et al. 2018; Swisher et al. 2018).

Although Lso has been a problem primarily in potatoes, the pathogen also infects peppers and other cultivated and wild plants primarily of the Solanaceae family (Butler and Trumble 2012; Goolsby et al. 2012; Thinakaran et al. 2015; Vereijssen et al. 2018). Under field conditions, various solanaceous plant species are often grown in the same vicinity, thereby increasing the probability that one species could be a source of infectious pathogens for another. Thus, knowledge of potential interactions between Lso haplotypes and different solanaceous crops is important in devising successful management strategies. The current project was initiated to (i) investigate host-pathogen interactions between Lso haplotypes and different cultivated solanaceous hosts, (ii) determine reactions of different 
pepper cultivars to haplotypes of Lso, and (iii) quantify the relative incidence of individual A and B haplotypes in plants after infestation by psyllids carrying both haplotypes (AB).

\section{Materials and Methods}

Psyllid colonies. Colonies of psyllids carrying a mix of both Lso haplotypes $(\mathrm{AB})$, originally collected from Edinburg in South Texas, have been maintained in our greenhouse since 2010. The Plant Pathology greenhouse temperature routinely rises above the optimum for psyllid reproduction during the summer, often resulting in dwindling psyllid numbers. Thus, the colonies have been regularly replenished with psyllids from other laboratories in order to have enough psyllids for multiple projects. Psyllid colonies carrying Lso AB were obtained from Dr. Badillo's laboratory in Weslaco, Texas, multiple times over the last several years. Psyllids carrying Lso A were obtained from Dr. Tamborindeguy's laboratory in College Station, Texas, and those carrying Lso B were obtained by letting Lso-free psyllids feed on potato plants identified to be infected only with Lso B. The psyllids (all Central haplotype) were reared in cages on eggplants, potatoes, and/or tomatoes and tested routinely as described below to verify that individual Lso haplotypes were true to type. Multiple cages of individual psyllids carrying the different Lso haplotypes are maintained in the greenhouse, and the different colonies are not used in new projects unless they test $100 \%$ true to type.

Host and Lso haplotype interactions. In 2017, jalapeno peppers (cultivar Am13sp11), potatoes (cultivar FL 1867), and tomatoes (cultivar Lance) were planted in 15-cm-diameter pots in the greenhouse in cages (two plants per cage, Bugdorms; BioQuip, Rancho Dominguez, CA), with two cages per treatment serving as replicates. Cages were laid out on benches in a randomized complete block design. At flower-bud initiation, plants in each cage were infested with psyllids (Central psyllid haplotype, six psyllids per plant) carrying Lso haplotype A, B, or AB or with Lso-negative $(\mathrm{N})$ psyllids where the psyllids were placed under each plant in 50-ml Falcon tubes. Plants in noninfested cages served as controls.

Average greenhouse temperatures during the experiment ranged between $18.9 \pm 0.07^{\circ} \mathrm{C}$ (night) and $23.1 \pm 0.07^{\circ} \mathrm{C}$ (day) (HOBO data logger; Onset Computer, Bourne, MA). Approximately 7 weeks after infestation, samples (the top youngest leaves with stems attached) were collected from individual plants and stored at $-80^{\circ} \mathrm{C}$ until molecular tests were conducted. To determine the impact of host-Lso interactions on psyllid survival/reproduction, adult psyllids in each cage were aspirated into 50-ml Falcon tubes and counted.

The experiment was similarly repeated in 2018 in three replications per treatment. However, for counting psyllids, plants in each cage were shaken vigorously and consistently back and forth three times to release psyllids from the plants. Psyllids that were consequently observed on the walls of the cages were then counted. This was conducted two times on consecutive days and the average of the two counts was used as an estimate of relative numbers of psyllids in each cage. Average greenhouse temperatures ranged between $18.5 \pm 0.04^{\circ} \mathrm{C}$ (night) and $20.6^{\circ} \mathrm{C} \pm 0.05$ (day).

Interactions between pepper lines and Lso haplotypes. Six pepper breeding lines from the Texas A\&M AgriLife pepper breeding program were planted in the greenhouse in 15 -cm-diameter pots in cages (two plants per cage) and infested with psyllids carrying haplotype A, $\mathrm{AB}, \mathrm{B}$, or $\mathrm{N}$ at flower-bud initiation as above. Plants in uninfested cages served as controls. Treatments were arranged on greenhouse benches in a randomized complete block design in three replications. Average greenhouse temperatures during the experiment ranged between $22.1 \pm$ $0.10^{\circ} \mathrm{C}$ (night) and $25.7 \pm 0.09^{\circ} \mathrm{C}$ (day). Approximately 7 weeks after infestation, individual plants in each cage were sampled and stored as described above until molecular tests were conducted. Numbers of psyllids in each infested cage were also estimated by shaking plants and counting the psyllids that landed on the cage walls as described earlier.

In addition, in a separate experiment, two commercial bell pepper cultivars (Golden bell and Wonder bell) were infested at flower onset with psyllids carrying Lso haplotype $\mathrm{A}, \mathrm{AB}$, or $\mathrm{B}$ in three replications as described above. Plants were then sampled for Lso testing and the relative numbers of adult psyllids in each cage were estimated by shaking plants and counting the psyllids that landed on the cage walls as described above.

Quantification of Lso A and B haplotype incidence after infestation by psyllids. Plant samples collected from all three hosts ( 8 to 10 samples per host for the 2-year period) in Lso-host interaction tests (objective i) and verified to be positive for Lso were further haplotyped (see molecular tests below). This was done to investigate whether the $\mathrm{AB}$ haplotype mix separated into individual $\mathrm{A}$ and $\mathrm{B}$ in plant tissues and to determine their relative incidence.

Molecular tests. Tests for the detection of Lso in psyllids or plant samples were conducted as described by Wen et al. $(2009,2013)$ and Workneh et al. (2018). In brief, the commercial product QuickExtract Plant DNA Extraction Solution (Epicentre, San Diego, CA) was used to extract total DNA from psyllids, with a modified standard protocol. Psyllids were placed individually in 2-ml screw-cap tubes containing a $2.5-\mathrm{mm}$ stainless steel bead. The tubes were then dipped in liquid nitrogen for approximately $2 \mathrm{~min}$ and then ground using a VWR Hard Tissue Grinder (Sugar Land, TX). Thereafter, $100 \mu 1$ of the QuickExtract DNA solution was added to the tubes, quickly vortexed, and spun down. The tubes were then placed on a digital heat block at $65^{\circ} \mathrm{C}$ for $15 \mathrm{~min}$, followed by incubation on another digital heat block at $98^{\circ} \mathrm{C}$ for $5 \mathrm{~min}$. The DNA was then ready to use for quantitative real-time PCR tests. Plant tissue DNA extraction followed the DNeasy Plant Mini Kit (Qiagen) protocol with a slightly modified method. Plant tissue $(100 \mathrm{mg})$ was collected and put in 2$\mathrm{ml}$ screw-cap tubes containing a 5.0-mm stainless steel bead. Tubes were then dipped in liquid nitrogen for approximately $2 \mathrm{~min}$ and then ground using a VWR Hard Tissue Grinder. The final DNA sample was eluted in $100 \mu$ l of the elution buffer.

After DNA extractions, an absolute quantification method was employed using an ABI ViiA 7 Real-Time PCR machine to detect Lso in plant samples or psyllids. The reaction mix consisted of TaqMan Fast Advanced Master Mix (Applied Biosystems), $0.3 \mu \mathrm{M}$ of forward primer Lso F (Li et al. 2009), $0.3 \mu \mathrm{M}$ of reverse primer $\mathrm{HLBr}$ (Li et al. 2006), and $0.25 \mu \mathrm{M}$ of HLBp TaqMan probe (Li et al. 2006). The PCR run method was adopted from the default run method designed by the ABI ViiA 7 system and consisted of one cycle of $20 \mathrm{~s}$ at $95^{\circ} \mathrm{C}, 40$ cycles of $1 \mathrm{~s}$ at $95^{\circ} \mathrm{C}$, and $20 \mathrm{~s}$ at $60^{\circ} \mathrm{C}$. If a sample tested positive for Lso, it was then analyzed to determine the Lso haplotype.

Lso haplotypes were determined on an ABI ViiA 7 Real-Time PCR unit. Lso A and Lso B were tested separately by using two sets of primer pairs and master mix. Each reaction mix of $20 \mu \mathrm{l}$ consisted of $1 \times$ SYBR Select Master Mix, $0.25 \mu \mathrm{M}$ of each of the forward and reverse primers (see below), and $2 \mu$ l of template DNA. The forward primer LsoHA6f (5'-GGT ACG AGC AAG CAT AAG CAA-3') and the reverse primer LsoHA6r (5'-AGC AAC CAA TTT TAC GGG AGT- $3^{\prime}$ ) were added to the reaction mix to detect Lso A. The forward primer LsoHB6f ( $5^{\prime}$-ACG GCA TTC ACT ATC GCT TT- $\left.3^{\prime}\right)$ and the reverse primer LsoHB6r (5'-CGC TCT CTA CGC CTC TCT CT-3') were added to the reaction mix to detect Lso haplotype $\mathrm{B}$. The mixes were prepared separately as a singleplex PCR for each haplotype detection. SYBR Green analysis was performed in three stages. The initial stage included enzyme activation at $50^{\circ} \mathrm{C}$ for $2 \mathrm{~min}$, followed by $10 \mathrm{~min}$ at $95^{\circ} \mathrm{C}$. The quantitative PCR (qPCR) stage was set to $40 \mathrm{cy}-$ cles of denaturing at $95^{\circ} \mathrm{C}$ for $15 \mathrm{~s}$ and annealing and extension at $60^{\circ} \mathrm{C}$ for $1 \mathrm{~min}$. The melting (dissociation) stage was included for $15 \mathrm{~s}$ at $95^{\circ} \mathrm{C}, 1 \mathrm{~min}$ at $60^{\circ} \mathrm{C}$ for annealing, and $15 \mathrm{~s}$ at $95^{\circ} \mathrm{C}$ for melting.

In addition to the above SYBR Green analysis, one of the several primer pairs (Lso-SSR-1F and Lso-SSR-1R) used to distinguish between Lso A and Lso B, as described by Lin et al. (2012) and RojasMartinez et al. (2016), was used to determine whether these primers could distinguish between the two Lso haplotypes in pepper samples with mixed infections of both Lso A and B. The haplotypes in the infected pepper samples were originally verified with the two pairs of primers (LsoHA6f, LsoHA6r and LsoHB6f, LsoHB6r) using SYBR Green analysis, as described above. Pepper samples that were identified to be positive for Lso A, Lso B, or Lso AB were used for tests 
with Lso-SSR-1F and Lso-SSR-1R primers. In these tests, the conventional PCR amplification reaction mix was used, which consisted of a total of $20 \mu \mathrm{l}$, with $1 \times$ GoTaq buffer, $0.75 \mu \mathrm{M}$ of forward primer Lso-SSR 1F, $0.75 \mu \mathrm{M}$ of reverse primer Lso-SSR 1R, $2.0 \mathrm{mM}$ of $\mathrm{MgCl}_{2}$, and 1.5 units of GoTaq polymerase. The PCR reaction was performed on a PTC-200 Peltier Thermal Cycler, and the run profile consisted of one initial denaturing cycle for $5 \mathrm{~min}$ at $94^{\circ} \mathrm{C}$, followed by 35 cycles of $30 \mathrm{~s}$ at $94^{\circ} \mathrm{C}, 30 \mathrm{~s}$ at $58^{\circ} \mathrm{C}, 45 \mathrm{~s}$ at $72^{\circ} \mathrm{C}$, and $10 \mathrm{~min}$ at $72^{\circ} \mathrm{C}$ for final extension. The PCR products were purified using the Qiagen MinElute PCR Purification Kit. The purified PCR products were then loaded on a $1.5 \%$ gel and the gel image was taken by a UVP MultiDoc-It Digital Imaging System device.

Data analyses. All data analyses were conducted using SAS software (version 9.4; SAS Institute Inc., Cary, NC). Psyllid count data were transformed using $\log _{10}\left(+1 \times 10^{-3}\right)$ to meet the requirement of the assumptions of homogeneity of variances. A two-way analysis of variance (ANOVA) was conducted using PROC GLM in which psyllid count data were treated as dependent and host and haplotype (including their interactions) were treated as independent variables, followed by the Tukey mean separation tests.

\section{Results}

Host and Lso haplotype interaction. Combined ANOVA results of the 2-year experiments across replicates showed that there was no experiment-haplotype interaction $(P=0.6637)$. However, there was a highly significant interaction between Lso haplotypes and hosts $(P<0.0001)$. Thus, the impact of different Lso haplotype treatments on psyllid numbers was compared within individual hosts for each experiment (Fig. 1). For the first experiment, no adult psyllids were detected on peppers infested with psyllids carrying Lso A 7 weeks after infestations, and leaf samples from the infested plants tested negative for Lso. Inspection of the pepper plants revealed no eggs or nymphs in any of the cages infested with Lso A psyllids. Psyllids carrying Lso A did not survive on peppers. However, peppers infested with psyllids carrying Lso AB, B, or N had substantial numbers of psyllids and all infested plants tested positive for Lso (Fig. 1A). The differences between Lso A and the other Lso treatments in their impact on psyllid numbers was highly significant $(P<0.0001$; Fig. $1 \mathrm{~A})$. In potatoes and tomatoes, psyllids carrying each of the different Lso haplotype treatments, including psyllids carrying only Lso A, copiously reproduced and there was no significant effect of Lso treatments on psyllid counts (Fig. 1A). In addition, plant samples from all tomato and potato plants infested with bacteriliferous psyllids, regardless of haplotype, tested positive for Lso, whereas all plants infested with $\mathrm{N}$ tested negative.

The second Lso haplotype-host interaction experiment showed results very similar to that of the first, in which no Lso A-carrying psyllids survived or reproduced and no Lso was detected in infested pepper plants. An exception to this was that one psyllid was detected in one of the three cages (reps) of Lso A on the second count. The psyllid tested negative for Lso and we think that it probably survived but lost the Lso, which can happen because Lso is a facultative symbiont and psyllids can acquire and also lose it (Darby et al. 2001; Thao et al. 2000; Workneh et al. 2016). All plant samples from Lso A cages (including from a cage in which one psyllid was observed) tested negative for Lso (Fig. 1B). There were abundant psyllids (as in the first experiment) in pepper cages infested with Lso AB, $\mathrm{B}$, and $\mathrm{N}$, and leaf samples collected from the pepper plants tested positive for Lso, except those infested with N. As in the first experiment, Lso haplotype had no impact on psyllid numbers on potato and tomato plants, and psyllids reproduced abundantly in all cages. There was no significant difference in psyllid counts among Lso haplotype treatments, except in tomatoes where plants infested with Lsonegative psyllids had significantly higher numbers of psyllids than those infested with psyllids carrying Lso A (Fig. 1B).

Interaction between pepper cultivars and Lso haplotypes. Symptoms of the disease on all pepper cultivars infested with psyllids carrying Lso $\mathrm{B}$ and $\mathrm{AB}$ were similar to those observed on potatoes (bushiness on top branches) but were much less severe than those in potatoes. Leaf variegations reported elsewhere (Camacho-Tapia et al. 2011) were not observed, but there was excessive dropping of leaves. However, there were no leaf symptoms and there was no excessive leaf dropping in pepper plants infested with psyllids carrying Lso A.

In the first study with six jalapeno breeding lines, there was no cultivar-haplotype interaction. As in the host-Lso haplotype interaction tests, psyllids carrying Lso A did not reproduce on any of the six lines of peppers (Fig. 2; Table 1), and leaf samples from infested plants tested negative for Lso. However, all pepper lines infested with psyllids carrying Lso $\mathrm{AB}$ and $\mathrm{B}$ and Lso-negative psyllids had variable but abundant psyllid numbers (Fig. 2; Table 1), and samples from the plants tested positive for Lso except those from plants infested with psyllids without Lso.

Tests with the bell pepper cultivars provided similar results as those obtained with the breeding lines. Psyllids carrying Lso A did not reproduce on either cultivar and plant samples tested negative for Lso. In addition, psyllids carrying Lso AB and $\mathrm{B}$ reproduced well on both bell pepper cultivars and samples from the plants tested positive for Lso (Fig. 2; Table 1). Abacteriliferous psyllids (N) were not included in this test.

Quantification of Lso A and B haplotypes after psyllid infestation. In all Lso haplotype-host interaction tests, one of the treatments was host infestation with individual psyllids carrying Lso AB. In leaf samples from these hosts, both Lso A and B were
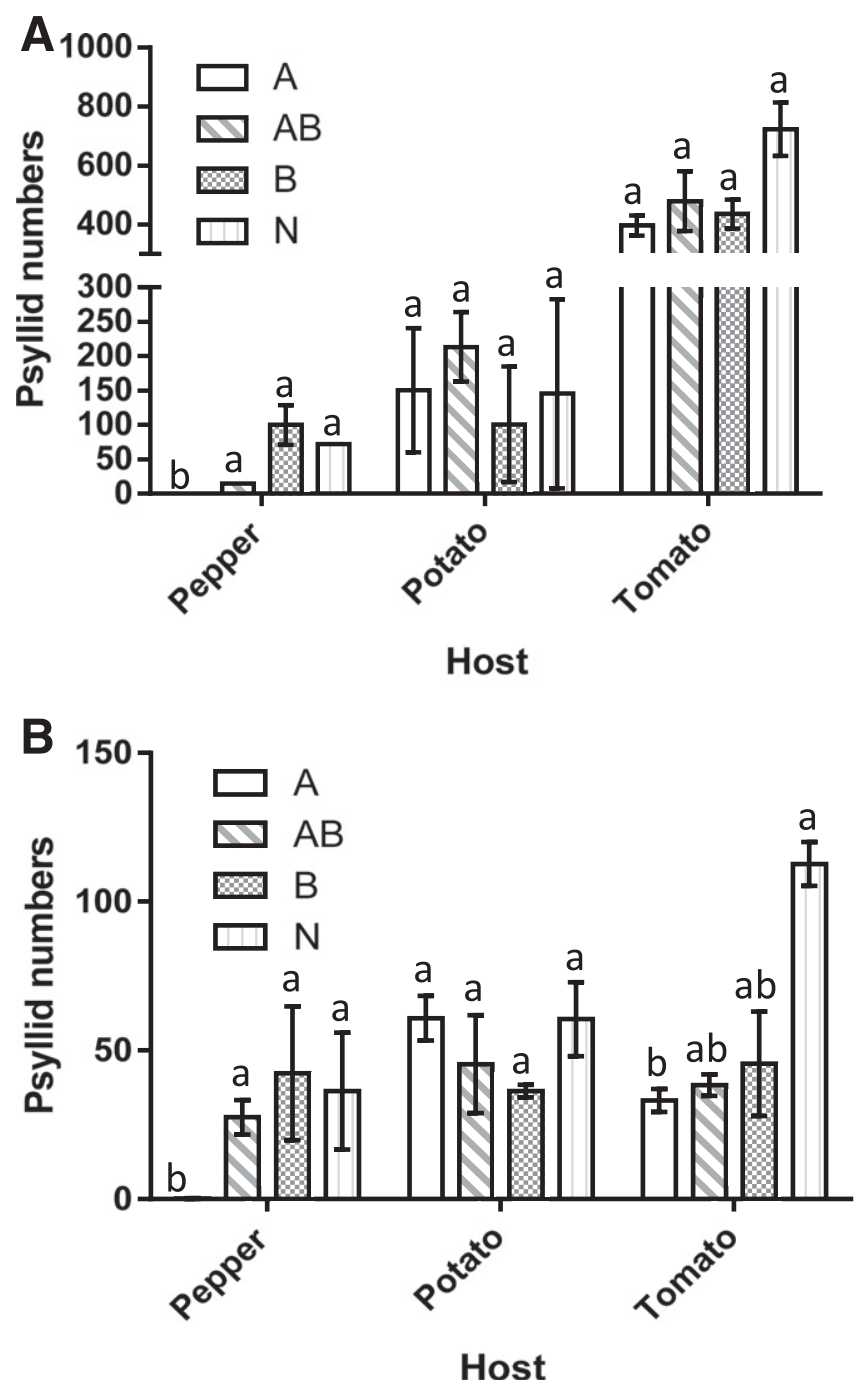

Fig. 1. Mean psyllid numbers on peppers, potatoes, and tomatoes each infested with psyllids carrying 'Candidatus Liberibacter solanacearum' (Lso) haplotype A, AB, or B or with Lso-free psyllids $(\mathrm{N})$ for the $\mathbf{A}$, first and $\mathbf{B}$, second experiments. Bars with the same letter are not significantly different based on Tukey's mean separation test $(P>0.05)$. 
detected either individually or as an $\mathrm{AB}$ mix. In peppers, haplotype A was detected in a significantly large majority of the samples (Fig. 3), capturing nearly 88\%; however, haplotype B was not detected singly but only as an $\mathrm{AB}$ mix, which comprised a small

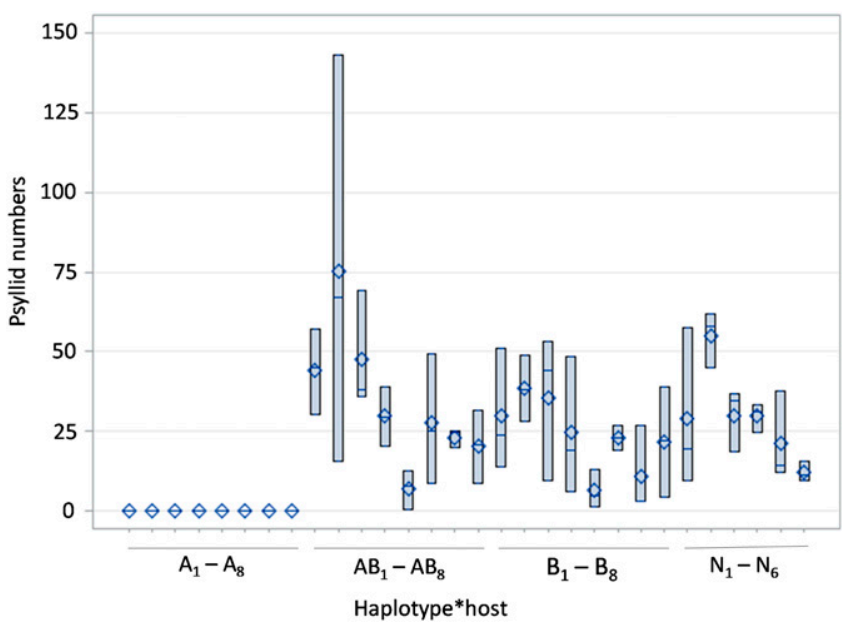

Fig. 2. Distribution of counts of psyllids as affected by haplotype-host (pepper cultivars) interactions.

Table 1. Reactions of pepper lines infested with psyllids carrying different 'Candidatus Liberibacter solanacearum' (Lso) haplotypes and Lso-negative psyllids and estimated mean numbers of psyllids on infested plants

\begin{tabular}{|c|c|c|c|}
\hline Cultivarw & Lso haplotype $^{x}$ & Tests for $\mathbf{L s o}^{y}$ & Psyllids $(n)^{\mathbf{z}}$ \\
\hline \multirow[t]{4}{*}{1} & A & - & $0.0 \pm 0.0$ \\
\hline & $\mathrm{AB}$ & + & $44.2 \pm 7.7$ \\
\hline & B & + & $29.7 \pm 11.1$ \\
\hline & $\mathrm{N}$ & - & $28.8 \pm 14.6$ \\
\hline \multirow[t]{4}{*}{2} & A & - & $0.0 \pm 0.0$ \\
\hline & $\mathrm{AB}$ & + & $75.2 \pm 37.0$ \\
\hline & B & + & $38.3 \pm 6.1$ \\
\hline & $\mathrm{N}$ & - & $55.0 \pm 5.1$ \\
\hline \multirow[t]{4}{*}{3} & A & - & $0.0 \pm 0.0$ \\
\hline & $\mathrm{AB}$ & + & $47.7 \pm 10.7$ \\
\hline & B & + & $35.5 \pm 13.3$ \\
\hline & $\mathrm{N}$ & - & $30.0 \pm 5.8$ \\
\hline \multirow[t]{4}{*}{4} & A & - & $0.0 \pm 0.0$ \\
\hline & $\mathrm{AB}$ & + & $29.7 \pm 5.3$ \\
\hline & B & + & $24.5 \pm 12.6$ \\
\hline & $\mathrm{N}$ & - & $29.7 \pm 2.7$ \\
\hline \multirow[t]{4}{*}{5} & A & - & $0.0 \pm 0.0$ \\
\hline & $\mathrm{AB}$ & + & $7.0 \pm 3.5$ \\
\hline & B & + & $6.5 \pm 3.4$ \\
\hline & $\mathrm{N}$ & - & $21.3 \pm 8.1$ \\
\hline \multirow[t]{4}{*}{6} & A & - & $0.0 \pm 0.0$ \\
\hline & $\mathrm{AB}$ & + & $27.7 \pm 11.9$ \\
\hline & B & + & $23.0 \pm 4.0$ \\
\hline & $\mathrm{N}$ & - & $12.2 \pm 1.8$ \\
\hline \multirow[t]{3}{*}{7} & A & - & $0.0 \pm 0.0$ \\
\hline & $\mathrm{AB}$ & + & $23.2 \pm 1.6$ \\
\hline & B & + & $11.0 \pm 8.0$ \\
\hline \multirow[t]{3}{*}{8} & A & - & $0.0 \pm 0.0$ \\
\hline & $\mathrm{AB}$ & + & $20.3 \pm 6.7$ \\
\hline & B & + & $21.8 \pm 9.9$ \\
\hline
\end{tabular}

w Cultivar numbers 1 to 6 represent breeding lines, where $1=$ Anaheim, $2=$ Cayenne, 3 = Jalapeno, $4=$ Aji (Capsicum baccatum), $5=$ Habanero, and 6 = Jalapeno (cultivar AM13sps11, which was also used in host-haplotype interaction tests). Cultivars 7 and 8 are commercial bell peppers (Golden bell and Wonder bell, respectively) obtained from a store.

x $\mathrm{AB}=$ individual psyllid carrying both Lso $\mathrm{A}$ and Lso $\mathrm{B}$ and $\mathrm{N}=$ Lsonegative psyllid.

y Minus and plus signs indicate negative and positive results, respectively.

$\mathrm{z}$ Values are presented as means \pm SEM. fraction of the pepper samples (12\%), for which the difference was highly significant $(P=0.0022)$. However, in potatoes and tomatoes, there was no significant difference in the frequencies of the haplotypes although the $\mathrm{AB}$ mix was numerically greater in both potato and tomato hosts, whereas B by itself, as in peppers, was not detected in tomatoes (Fig. 3). Overall, the AB mix mostly stayed intact in potato and tomato tissues but the haplotype mix was replaced in large part by A in peppers.

Results of testing pepper samples with mixed infections are presented in Figure 4. The two primer pairs (LsoHA6f, LsoHA6r and LsoHB6f, LsoHB6r) used in the SYBR Green analysis, clearly distinguished between Lso A and Lso B in both single (Fig. 4A and B) and mixed infections (Fig. 4C, D, and E). However, amplification results using Lso-SSR-1F and Lso-SSR-1R depended on the titer levels of the individual Lso haplotypes in the mixed infections. In samples in which Lso A had a greater titer level than Lso B, only Lso A was amplified and vice versa. The primers amplified both Lso A and Lso B only when the two haplotypes had similar titer levels. For example, when Lso A had a cycle threshold (CT) value of 22 and Lso B had a CT value of 28, only Lso A was amplified (Fig. 5, lane C). Similarly, Lso B with a CT value of 25 was amplified but Lso A with a CT value of 30 was not (Fig. 5, lane D). However, in a sample in which both haplotypes had similar CT values of 23 each, both haplotypes were amplified (Fig. 5, lane E).

\section{Discussion}

This 2-year project investigated the relationships between three solanaceous hosts (peppers, potatoes, and tomatoes) and psyllids carrying Lso haplotypes (A, B, and AB) as well as Lso-negative psyllids in terms of infection by Lso and psyllid abundance on individual hosts. Overall, the investigation produced several novel results. The finding that psyllids carrying only Lso A could not survive on peppers while Lso-negative psyllids abundantly reproduced singles out Lso A as the sole responsible factor for the outcome. The fact that samples from plants infested with psyllids carrying Lso A tested negative is interesting and important, but the most surprising finding in this project was the association of Lso A with a lack of psyllid survival on peppers but not with potatoes or tomatoes. The mechanism behind this is currently not understood. We conducted repeated searches in Lso A cages throughout the duration of this study and could not come up with any eggs or nymphs. It is possible that either the psyllids did not lay eggs or could not feed and starved to death. It is also possible that the psyllids lived out their days without

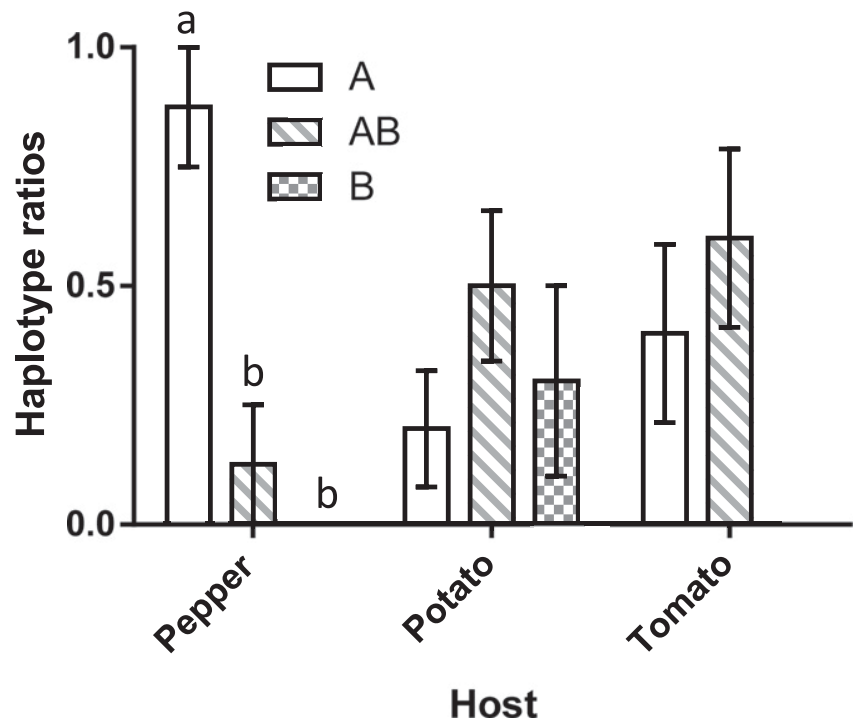

Fig. 3. Relative incidence of 'Candidatus Liberibacter solanacearum' (Lso) haplotypes $(A, A B$, and $B)$ detected in plant samples after host (pepper, potato, and tomato) infestation with individual psyllids carrying both $A$ and $B$ haplotypes. Bars with the same letter are not significantly different based on Tukey's mean separation test $(P>0.05)$. 
reproducing. The failure to detect Lso in pepper plants after infestation by psyllids carrying only Lso A also may suggest that either these psyllids did not effectively probe or they probed and transmitted the pathogen into plant tissues but Lso A could not initiate a successful infection.

The cultivar-Lso haplotype interaction objective was initiated following results from the original haplotype-host interaction tests in which psyllids carrying Lso A did not survive on peppers and samples from infested plants tested negative. The primary objective was to further investigate whether cultivar could be a factor in inhibiting psyllid reproduction and infection by Lso. Based on the results of this study, we now conclude that pepper genotype was not a factor because all eight lines with different genetic backgrounds provided similar results.

Quantification of Lso A and Lso B in plants infested with psyllids carrying Lso AB provided additional evidence that Lso A can infect and be detected in peppers only when it is associated with Lso B. It appears that B is not only important for Lso A to infect peppers but also counteracts the deleterious effect of Lso A on psyllid survival. This implies that Lso A requires B to have any epidemiological impact in peppers. Apparently, once Lso A infects a pepper plant, it can outcompete $\mathrm{B}$ after infestation by $\mathrm{AB}$ psyllids, as evidenced by greater frequencies of detection and a total absence of Lso B in the infected plant. However, on occasion, we have observed haplotype B to be the dominant haplotype in infected pepper plants after infestation by AB psyllids. Preliminary studies (Workneh et al., unpublished data) indicate that temperature may affect which haplotype dominates in mixed pepper plant infections. Yet the finding in this study that successful infections of pepper by Lso A only occurred when plants initially were infested with psyllids carrying Lso $\mathrm{AB}$ was both significant and novel.

Psyllid counts in the first haplotype-host interaction experiment were higher than those in the second experiment. This was not surprising because of the different methodology used to quantify psyllid numbers in the two experiments. In the first experiment, all adult psyllids in each cage were aspirated and counted. However, this approach could not be sustained because of the time and labor it required. Thus, we adopted the method of plant shaking and counting the psyllids observed subsequently on the walls of the cages. This method was significantly faster, and there was no experiment-haplotype interaction in the analysis of psyllid counts, indicating that the changes in counting methodology had no significant effect on final results.

Lso was initially identified in New Zealand from diseased solanaceous crops (Liefting et al. 2008, 2009); to date, Lso A is the only haplotype that has been reported in the country (Nelson et al. 2011; Thompson et al. 2015). Because pepper was one of the hosts from which Lso was first described, the implication is that Lso A can infect peppers in New Zealand in the absence of Lso B, which contradicts the results of this study. It is possible that different cultivars or environmental conditions (e.g., temperature) between the two locations may have played a significant role in contributing to the different results in infection of peppers by Lso A. However, Thompson et al. (2015) described several genetic differences between isolates of Lso A from the United States and New Zealand. In qPCR analysis, they identified considerable within-haplotype diversity and concluded that significant genome plasticity exists in the species. They described the differences in genome arrangements and prophage locations, which they said suggest locus diversity within the haplotypes. Noting reported differences in zebra chip symptom expression in infected potato tubers from the United States and New Zealand, and even within the United States, Thompson et al. (2015) stated that disease symptom variability may occur not only between haplotypes but within haplotypes as well. Thus, we believe that potential differences in the ability of Lso haplotype A to infect peppers in the United States and New Zealand may result from genetic variability within haplotype A strains. A similar genetic variation within haplotype $\mathrm{C}$ detected in different plant species was also reported (Haapalainen et al. 2018). Clearly, the issue of genetic variation and subsequent biological differences among and within Lso haplotype strains deserves further investigation.

Lso A was also reported to be detected in peppers in Mexico (Rojas-Martinez et al. 2016; Swisher et al. 2018). Those strains of Lso A may have been different, as discussed above, that were able to infect peppers in the absence of Lso B. However, in addition to $\mathrm{A}$, mixes of $\mathrm{AB}$ haplotypes were detected in surveys conducted by

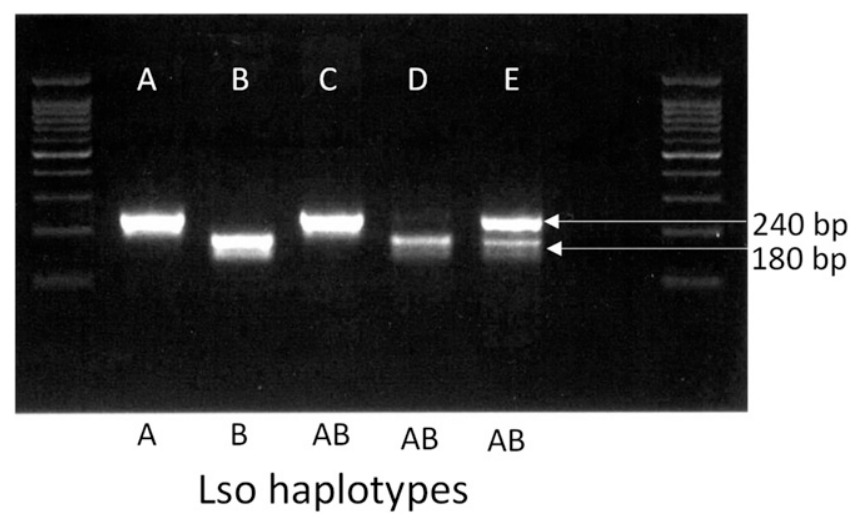

Fig. 5. Agarose gel PCR product amplified with 'Candidatus Liberibacter solanacearum' (Lso) forward and reverse Lso-SSR-1F and Lso SSR-1R primers from the same pepper samples used in Figure 4. Amplicons in lanes $A$ to $E$ correspond to the respective samples used for graphs in Figure $4 \mathrm{~A}$ to $\mathrm{E}$.

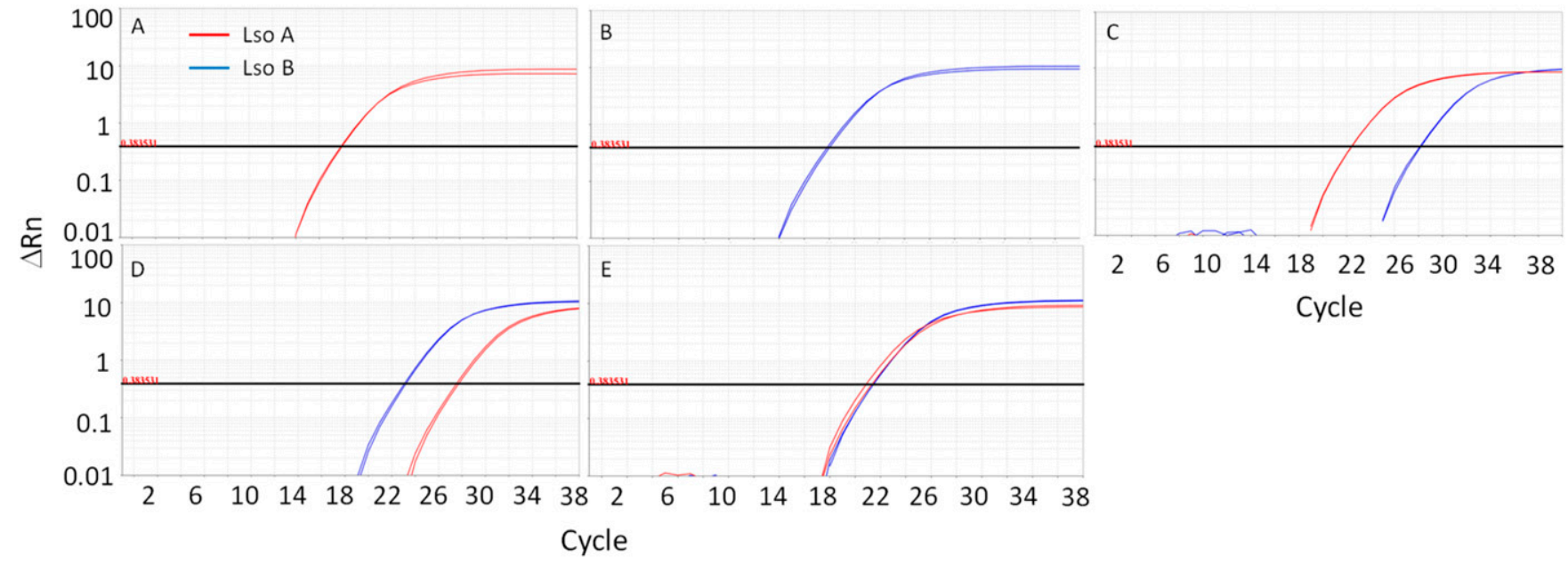

Fig. 4. Amplification plots of 'Candidatus Liberibacter solanacearum' (Lso) haplotypes in pepper samples infected with A, Lso A, B, Lso B, and C, D, and E, AB mixes using two pairs of primers (LsoHA6f, LsoHA6r and LsoHB6f, LsoHB6r). $\triangle$ Rn represents the normalized reporter value, which stands for changes in fluorescent signal with increasing CT values. 
Swisher et al. (2018), suggesting that infection could have occurred with psyllids carrying $\mathrm{AB}$ mixes, which supports our view. However, Rojas-Martinez et al. (2016) did not detect AB mixes in 200 plant and psyllid samples. It is possible that those pepper plants may have also been infected with $\mathrm{AB}$ mixes, but one haplotype predominated in the infection and only the dominant one was detected. Since both haplotypes A and B were individually detected at the locations, we found it peculiar that the authors never identified $\mathrm{AB}$ mixes in any of the plant or psyllid samples they tested. In order to elucidate possible reasons for the different results reported in their study and ours, we used their primers (Lso-SSR-1F and Lso-SSR-1R) on plant samples from our laboratory that were known to be positive for mixed $\mathrm{AB}$ infections. In some of our mixed Lso AB samples, Lso A titer was higher than that of B; and in some samples, Lso B titer was higher. However, when we used the methods of Rojas-Martinez et al. (2016), only the Lso haplotype with the highest titer was detected and the lowtiter haplotype in the mixed infections was not detected. The primers detected both haplotypes in mixed infections only when their titer levels were similar (Fig. 5, lanes C to E). Amplifications of only the predominant isolate in mixed infections using conventional PCR (with one primer pair) were also described previously for sugar beet viruses (Rush et al. 1994). However, with the techniques used in our laboratory for Lso haplotype detection and identification, which are described in this article, we were able to clearly identify both haplotypes in the exact same mixed samples (Fig. 4). Thus, it is entirely possible, and even probable, that Lso A detected in peppers by RojasMartinez et al. (2016) may have been from mixed infections.

In conclusion, in this study, pepper plants infested by psyllids carrying only Lso A did not become infected, but tomato and pepper plants infested with psyllids from the same colony were infected and developed disease symptoms. More surprisingly, psyllids carrying only Lso A were unable to survive on peppers but psyllids carrying Lso AB were abundantly observed. Once identified, the mechanisms involved in affecting the survival of psyllids carrying Lso A on peppers or the mechanisms preventing infection of peppers by Lso A could have significant implications for psyllid management and control of zebra chip.

\section{Acknowledgments}

We acknowledge technical support from J. Arthur, J. Bull, J. Gray, and Jordan Trees. We are grateful to Dr. Kevin Crosby (Horticulture Department, College Station, TX) for providing us with seeds for pepper lines. We thank Dr. Cecilia Tamborindeguy (Texas A\&M, College Station) for providing us with psyllids carrying Lso haplotype A.

\section{Literature Cited}

Alfaro-Fernández, A., Cebrian, M. C., Villaecusa, F. J., Hermosa de Mendoza, A., Ferrandiz, J. C., Sanjuan, S., and Font, M. I. 2012a. First report of 'Candidatus Liberibacter solanacearum' in carrot in mainland Spain. Plant Dis. 96:582.

Alfaro-Fernández, A., Severio, F., Cebrian, M. C., Villaecusa, F. J., and Font, M. I. 2012b. 'Candidatus Liberibacter solanacearum' associated with Bactericera trigonica-affected carrots in Canary Islands. Plant Dis. 96:581.

Butler, C. D., and Trumble, J. T. 2012. The potato psyllid, Bactericera cockerelli (Sulc) (Hemptera: Triozidae): Life history relationship to plant diseases, and management strategies. Terr. Arthropod Rev. 5:87-111.

Camacho-Tapia, M., Rojas-Martinez, R. I., Zavaleta-Mejia, E., Herandez-Deheza, M. G., Carrilloo-Salazar, J. A., Rebollar-Alviter, A., and Ochoa-Martinez, D. L. 2011. Aetiology of chili pepper variegation from Yurecuaro, Mexico. J. Plant Pathol. 93:331-335.

Crosslin, J. M., Hamm, P. B., Eggers, J. E., Rondon, S. I., Sengoda, V. G., and Munyaneza, J. E. 2012a. First report of zebra chip disease and 'Candidatus Liberibacter solanacearum' on potatoes in Oregon and Washington State. Plant Dis. 96:452.

Crosslin, J. M., Olsen, N., and Nolte, P. 2012b. First report of zebra chip disease and 'Candidatus Liberibacter solanacearum' on potatoes in Idaho. Plant Dis. 96:453.

Darby, A. C., Birkle, L. M., Turner, S. L., and Douglas, A. E. 2001. An aphidborne bacterium allied to the secondary symbionts of whitefly. FEMS Microbiol. Ecol. 36:43-50.

European and Mediterranean Plant Protection Organization. 2012. First report of 'Candidatus Liberibacter solanacearum' on carrots and celery in Spain in association with Bactericera trigonica. EPPO Rep. Serv. Pests Dis. 6:4-5.

Goolsby, J. A., Adamczyk, J. J., Crosslin, J. M., Toxclair, N. N., Anciso, J. R., Bester, G. G., Bradshaw, J. D., Bynum, E. D., Carpio, L. A., Henne, D. C., Joshi, A., Munyaneza, J. E., Porter, P., Sloderbeck, P. E., Supak, J. R., Rush,
C. M., Willet, F. J., Zachmann, B. J., and Zens, B. A. 2012. Seasonal population dynamics of potato psyllid (Hemiptera: Triozidae) and its associated pathogen 'Candidatus Liberibacter solanacearum' in potatoes in the southern Great Plains of North America. J. Econ. Entomol. 105:1268-1276.

Haapalainen, M., Wang, J., Latvala, S., Lehtonen, M. T., Pirhonen, M., and Nissinen, A. I. 2018. Genetic variation of 'Candidatus Liberibacter solanacearum' haplotype $\mathrm{C}$ and identification of a novel haplotype from Trioza urticae and stinging nettle. Phytopathology 108:925-934.

Hansen, A. K., Trumble, J. T., Stouthamer, R., and Paine, T. D. 2008. A new huanglongbing species, 'Candidatus Liberibacter psyllaurous', found to infect tomato and potato, is vectored by the psyllid Bactericera cockerelli (Sulc). Appl. Environ. Microbiol. 74:5862-5865.

Li, W., Abad, J. A., French-Monar, R. D., Rascoe, J., Wen, A., Gudmestad, N. C. Secor, G. A., Lee, I., Duan, Y., and Levy, L. 2009. Multiplex real-time PCR for detection, identification and quantification of 'Candidatus Liberibacter solanacearum' in potato plants with zebra chip. J. Microbiol. Methods 78: 59-65.

Li, W., Hartung, J. S., and Levy, L. 2006. Quantitative real-time PCR for detection and identification of Candidatus Liberibacter species associated with citrus huanglongbin. J. Microbiol. Methods 66:104-115.

Liefting, L. W., Perez-Egusquia, Z. C., Clover, G. R. G., and Anderson, J. A. D. 2008. A new 'Candidatus' Liberibacter species in Solanum tuberosum in New Zealand. Plant Dis. 92:1474.

Liefting, L. W., Weir, B. S., Pennycook, S. R., and Clover, R. G. 2009 'Candidatus Liberibacter solanacearum' associated with plants in the family of Solanaceae. Int. J. Syst. Evol. Microbiol. 59:2274-2276.

Lin, H. and Gudmestad, N. C. 2013. Aspects of pathogen genomics, diversity, epidemiology, vector dynamics, and disease management for a newly emerged disease of potato: Zebra chip. Phytopathology 103:524-537.

Lin, H., Islam, M. S., Bai, Y., Wen, A., Lan, S., Gudmestad, N. C., and Civerolo, E. L. 2012. Genetic diversity of 'Candidatus Liberibacter solanacearum' strains in the United States and Mexico revealed by simple sequence repeat markers. Eur. J. Plant Pathol. 132:297-308.

Mendoza-Herrera, A., Levy, J., Harrison, K., Yao, J., Ibanez, F., and Tamborindeguy, C. 2018. Infection by 'Candidatus Liberibacter solanacearum' haplotype A and B in Solanum lycopersicum 'Moneymaker'. Plant Dis. 102: 2009-2015.

Munyaneza, J. E., Crosslin, J. M., and Upton, J. E. 2007. Association of Bactericera cockerelli (Homoptera: Psyllidae) with "zebra chip," a new potato disease in southwestern United States and Mexico. J. Econ. Entomol. 100:656-663. J. Econ. Entomol. 100:656-663.

Munyaneza, J. E., Fisher, T. W., Sengoda, V. G., Garczynski, S. F., Nissinen, A., and Lemmetty, A. 2010. First report of "Candidatus Liberibacter solanacearum" associated with psyllid-affected carrots in Europe. Plant Dis. 94:639.

Munyaneza, J. E., Sengoda, V. G., Sandheium, H., and Meadow, R. 2012a. First report of 'Candidatus Liberibacter solanacearum' associated with psyllidaffected carrots in Norway. Plant Dis. 96:454.

Munyaneza, J. E., Sengoda, V. G., Stegmark, R., Arvidsson, A. K., Anderbrant, O., Yuvaraj, J. K., Ramert, B., and Nissnen, A. 2012b. First report of 'Candidatus Liberibacter solanacearum' associated with psyllid-affected carrots in Sweden. Plant Dis. 96:453.

Nelson, W. R., Fisher, T. W., and Munyaneza, J. E. 2011. Haplotypes of 'Candidatus Liberibacter solanacearum' suggest-long standing separation. Eur. J. Plant Pathol. 130:5-12.

Rashed, A., Wallis, C. M., Paetzold, L., Workneh, F., and Rush, C. M. 2013. Zebra chip disease and potato biochemistry: Tuber physiological changes in response to 'Candidatus Liberibacter solanacearum' infection over time. Phytopathology 103:419-426.

Rehman, M., Melgar, J. C., Rivera, J. M., Idris, A. M., and Brown, K. 2010. First report of 'Candidatus Liberibacter psyllaurous' or ' $\mathrm{Ca}$. Liberibacter solanacearum' associated with severe foliar chlorosis, curling, and necrosis and tuber discoloration of potato plants in Honduras. Plant Dis. 94:376.

Rojas-Martinez, R. I., Camacho-Tapia, M., Zavaleta-Mejia, E. and Levy, J. 2016. First report of the presence of haplotypes A and B of 'Candidatus Liberibacter solanacearum' in chili (Capsicum annuum L.) in the central region of Mexico. J. Plant. Path. 98:111-115.

Rosson, P. 2006. Economic Impacts of Zebra Chip on the Texas Potato Industry. Texas AgriLife Research Economists, Center for North American Studies, Texas A\&M University, College Station.

Rush, C. M., French, R., and Heidel, G. B. 1994. Differentiation of two closely related furoviruses using the polymerase chain reaction. Phytopathology 84 : 1366-1369.

Rush, C. M., Workneh, F., and Rashed, A. 2015. Significance and epidemiological aspects of late-season infections in the management of potato zebra chip. Phytopathology 105:929-936.

Secor, G. A., Lee, I. M., Bottner, K. D., Rivera, V., and Gudmestad, N. C. 2006 First report of a defect of processing potatoes in Texas and Nebraska associated with a new phytoplasma. Plant Dis. 90:377.

Secor, G. A., and Rivera, V. 2004. Emerging diseases of cultivated potato and their impact on Latin America. Rev. Latinoam. Papa 1(Suppl):1-8.

Swisher, K. D., Munyaneza, J. E., Velásquez-Valle, R., and Mena-Covarrubias, J. 2018. Detection of pathogens associated with psyllids and leafhoppers in 
Capsicum annuum L, in the Mexican states of Durango, Zacatecas, and Michoacán. Plant Dis. 102:146-153.

Swisher Grimm, K. D., and Garczynski, S. F. 2019. Identification of a new haplotype of 'Candidatus Liberibacter solanacearum' in Solanum tuberosum L. Plant Dis. 103:468-474.

Thao, M. L., Clark, M. A., Baumann, L., Brennan, E., Moran, N. A., and Baumann, P. 2000. Secondary endosymbionts of psyllids have been acquired multiple times. Curr. Microbiol. 41:300-304.

Thinakaran, J., Pierson, E., Kunta, M., Munyaneza, J. E., Rush, C. M., and Henne, D. C. 2015. Silverleaf nightshade (Solanum elaeagnofolium), a reservoir host for 'Candidatus Liberibacter solanacearum', the putative causal agent of zebra chip disease of potato. Plant Dis. 99:910-915.

Thompson, S. M., Johnson, C. P., Lu, A. Y., Frampton, R. A., Sullivan, K. L., Fiers, M. W. E. J., Crowhurst, R. N., Pitman, A. R., Scott, I. A. W., Wen, A., Gudmestad, N. C., and Smith, G. R. 2015. Genomes of 'Candidatus Liberibacter solanacearum' haplotype A from New Zealand and United States suggest significant genome plasticity in the species. Phytopathology 105:863-871.

Vereijssen, J., Smith, G. R., and Weintraub, P. G. 2018. Bactericera cockerelli (Hemiptera: Triozidae) and 'Candidatus Liberibacter solanacearum' in potatoes in New Zealand: Biology, transmission, and implications for management. J. Integr. Pest Manag. 9:1-21.

Wen, A., Johnson, C., and Gudmestad, N. C. 2013. Development of a PCR assay for the rapid detection and differentiation of 'Candidatus Liberibacter solanacearum' haplotypes and their spatiotemporal distribution in the United States. Am. J. Potato Res. 90:229-236.

Wen, A., Mallik, I., Alvarado, V. Y., Pasche, J. S., Wang, X., Li, W., Levi, L., Lin, H., Scholthoff, H. B., Mirkov, T. E., Rush, C. M., and Gudmestad, N. C. 2009 Detection, distribution, and genetic variability of 'Candidatus' Liberibacter species associated with zebra complex disease of potato in North America. Plant Dis. 93:1102-1115.

Workneh, F., Paetzold, L., Rashed, A., and Rush, C. M. 2016. Population dynamics of released potato psyllids and their bacteriliferous status in relation to disease incidence in caged field plots. Plant Dis. 100: 1762-1767.

Workneh, F., Paetzold, L., Silva, A., Johnson, C., Rashed, A., Badillo, I. Gudmestad, N. C., and Rush, C. M. 2018. Assessments of temporal variations in haplotypes of 'Candidatus Liberibacter solanacearum' and its vector the potato psyllid in potato fields and native vegetation. Environ. Entomol. 47:1184-1193. 